\title{
Metabolismo del glutatión y desarrollo posnatal del pulmón de la rata
}

\author{
Alejandro Ramírez ${ }^{1,2, a}$, Tatiana Reyes 1,2 , Leonel Liberona ${ }^{1,2, b}$, \\ Andrea Bustamente ${ }^{1,2}$, Claudia Sáez $2,3, c$, Gisella Borzone ${ }^{1,2}$. \\ Glutathione metabolism during \\ postnatal development of the rat lung
}

Background: Significant changes in lung antioxidants occur in preparation for birth. Little is known about physiological regulation of antioxidants in the postnatal period. Aim: To study the glutathione system in the lungs during postnatal development. Material and methods: In the lungs of 7, 15, 21, 50 and 70 days old SpragueDawley rats we measured total and oxidized glutathione content as well as the activity of the limiting enzyme in glutathione synthesis ( $\boldsymbol{\gamma}$ GCS) and of the enzymes glutathione peroxidase (GPx) and glutathione reductase (GRd). Results: Between 7 and 15 days the activities of GPx and GRd increase $32 \%$ and $26 \%$, respectively $(\mathrm{p}<0.001$ ). Whereas GPx activity remains high throughout the rest of the study period, GRd activity decreases progressively reaching adulthood values at 7 days. $\boldsymbol{\gamma}$ GCS activity shows a gradual increase that reaches significance at 50 days when it doubles values observed at 7 days $(\mathrm{p}<0.05)$. A significant correlation was found between GPx and GRd activities over the entire period ( $r=0.62, p<0.0001$ ). Strength of the correlation is age dependent due to the differences in time course of the enzyme changes. Whereas total GSH does not change, oxidized glutathione decreases from $7 \%$ at 7 and 15 days to $4 \%$ later on $(\mathrm{p}<0.01)$. Conclusions: The activity of several enzymes involved in glutathione metabolism increases during postnatal development of the rat lung. Interpretation of lung responses to injurious agents needs to be done taking into consideration the physiological regulation of antioxidants during postnatal development (Rev Méd Chile 2007; 135: 896-903).

(Key words: Antioxidants; Glutathione; Growth and development)

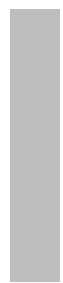

Recibido el 13 de septiembre, 2006. Aceptado el 28 de diciembre, 2006.

Financiado por Proyecto FONDECYT 1030412.

${ }^{1}$ Departamentos de Enfermedades Respiratorias y ${ }^{3}$ Hematología y ${ }^{2}$ Centro de Investigaciones Médicas, Facultad de Medicina, Pontificia Universidad Católica de Chile. Santiago, Chile.

aAlumno de Medicina, Pontificia Universidad Católica de Chile

bBioquímico

${ }^{\mathrm{c}} \mathrm{PhD}$

Correspondencia a: Dra. Gisella Borzone. Departamento de Enfermedades Respiratorias, Pontificia Universidad Católica de Chile. Marcoleta 352, Piso 1 (interior). Fonos: 354 8029354 3242. Fax: 6335255.

E mail: gborzone@med.puc.cl 
L a participación de las especies reactivas deriva das del oxígeno en enfermedades pulmonares ha sido estudiada durante años, ya que el pulmón es un órgano altamente expuesto a agentes oxidantes, como ocurre con el humo del cigarrillo, la contaminación ambiental y el uso de oxígeno en altas concentraciones ${ }^{1}$. Además, la acumulación de células inflamatorias en muchas enfermedades respiratorias, representa otra fuente importante de oxidantes $^{2}$. Sin embargo, la mayor parte de las investigaciones ha estado orientada a evaluar el papel dañino de los oxidantes ${ }^{3,4}$ y la información relacionada con la regulación o adaptación fisiológica de las defensas antioxidantes es limitada.

Esta regulación ha sido estudiada durante el período prenatal, tanto en seres humanos como en animales. Por un lado, estudios utilizando métodos inmunohistoquímicos en pulmones humanos muestran que las enzimas antioxidantes están presentes desde muy temprano en la vida intrauterina ${ }^{5}$. Por otra parte, otros investigadores han encontrado un aumento en la actividad de enzimas antioxidantes pulmonares en distintas especies de animales durante dicho período ${ }^{6-9}$. De acuerdo a Frank y cols, el aumento en la actividad de enzimas antioxidantes en la rata, se produce en el último 10\%-15\% del período gestacional, coincidiendo con la maduración del surfactante $^{7}$. Este aumento en defensas antioxidantes ha sido interpretado como una adaptación pulmonar para la exposición al ambiente relativamente hiperóxico que representa el nacimiento ${ }^{6}$.
A diferencia del período prenatal, la información sobre la regulación fisiológica de los antioxidantes pulmonares durante el período posnatal es escasa. Sólo el trabajo de Yam y cols aborda, en ratas, la regulación de algunos antioxidantes pulmonares en el período posnatal ${ }^{10}$. Tal vez por ello, ha sido menos aceptado el concepto de que los cambios en las defensas antioxidantes pulmonares que se inician al final de la gestación, pueden constituir el inicio de un proceso que se extiende al período posnatal y que se relaciona más con el desarrollo del pulmón que con el sólo proceso del nacimiento; particularmente, porque tanto en el ser humano como en animales, el desarrollo del pulmón no está completo al momento del nacimiento y la mayor parte del desarrollo alveolar es posnatal ${ }^{11-13}$.

Nuestro objetivo fue evaluar el metabolismo del glutatión (GSH) pulmonar durante el desarrollo posnatal de la rata. El sistema del glutatión es uno de los principales mecanismos antioxidantes del pulmón, ya que participa tanto a nivel celular como del líquido que cubre el epitelio alveolar2,14, protegiéndolo contra agentes oxidantes, compuestos electrofílicos y otros tóxicos. Forma parte de un ciclo de óxido-reducción ilustrado en la Figura 1, que es fundamental para prevenir la depleción de grupos-SH. En este ciclo, la reacción catalizada por la enzima glutatión peroxidasa (GPx) permite, a través de la oxidación del glutatión, la detoxificación del peróxido de hidrógeno y de distintos hidroperóxidos, mientras que

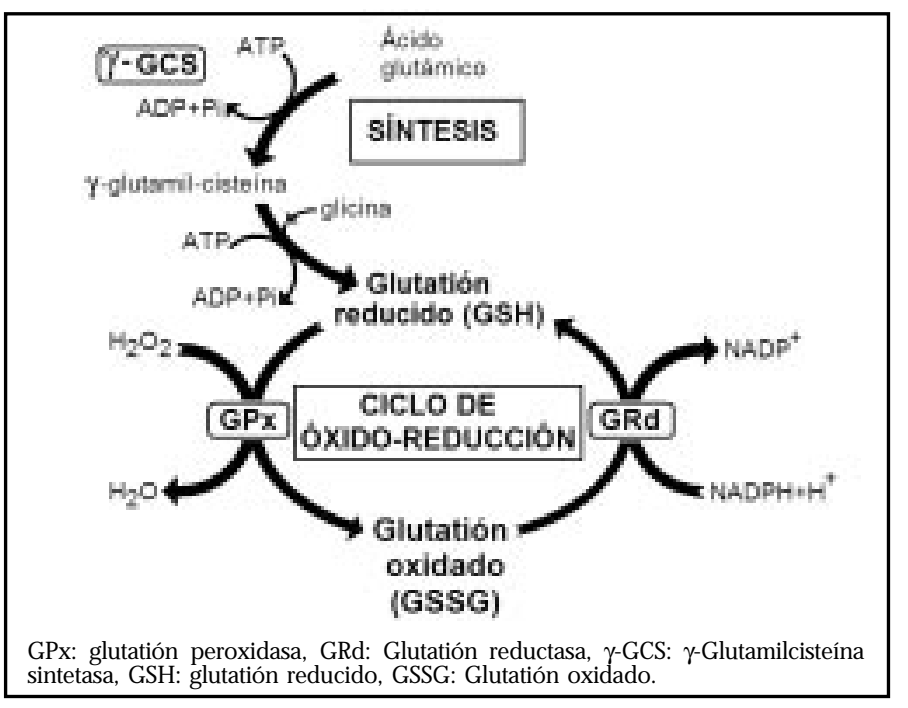

Figura 1. Síntesis y ciclo de óxido-reducción del glutatión. 
la enzima glutatión reductasa (GRd), cataliza la reducción del glutatión oxidado (GSSG), el que se mantiene en niveles cercanos a $1 \%$ del glutatión total in vivo ${ }^{2}$.

Por otra parte, la concentración intracelular de GSH, también depende del proceso de síntesis (Figura 1), en el que la enzima limitante es la $\gamma$ glutamilcisteína sintetasa $(\gamma \text {-GCS })^{2}$.

Para probar la hipótesis de que el desarrollo y crecimiento posnatal del pulmón va acompañado de cambios que afectan a distintas enzimas que participan en el metabolismo del glutatión, medimos el contenido de glutatión total y oxidado, así como la actividad de la enzima limitante de la síntesis y de las enzimas del ciclo de óxidoreducción del glutatión, en el pulmón de la rata, en el período posnatal entre los 7 y 70 días de vida.

\section{MATERIAL Y MÉTODO}

Estudiamos cinco grupos de ratas Sprague Dawley machos, alimentadas normalmente y mantenidas con un ciclo de luz-oscuridad de $12 \mathrm{~h}$. Escogimos edades representativas de distintas etapas de la vida posnatal, hasta la adultez: 7 días $(24+4 \mathrm{~g}, \mathrm{n}$ $=15), 15$ días $(26 \pm 3 \mathrm{~g}, \mathrm{n}=15), 21$ días $(54 \pm 6 \mathrm{~g}, \mathrm{n}$ =15), 50 días $(227 \pm 28 \mathrm{~g}, \mathrm{n}=15)$ y 70 días $(320 \pm 24$ $g, n=15)$. El estudio fue aprobado por el comité de ética de nuestra institución para trabajos en animales de experimentación.

Los animales fueron anestesiados con hidrato de cloral por vía intraperitoneal $(45 \mathrm{mg} / 100 \mathrm{~g}$ de peso) previo a la exsanguinación por sección de la vena cava inferior y extracción de ambos pulmones, utilizando técnicas de congelación con nitrógeno líquido. Los pulmones de animales de 50 y 70 días fueron divididos en tres segmentos y rotulados como superior, medio e inferior, previo al almacenamiento a $-80^{\circ} \mathrm{C}$, para estudiar la distribución de la actividad enzimática, mientras que los pulmones de animales de 7, 15 y 21 días, dado su pequeño tamaño, fueron almacenados sin dividir.

Medición de la actividad específica de las enzimas que participan en el metabolismo del glutatión. Se realizó en el homogeneizado del pulmón completo para los animales de 7,15 y 21 días de vida y en homogeneizados de cada uno de los tres fragmentos de pulmón de animales de 50 y 70 días. La actividad de la enzima GPx se midió con el método de Gunzler y cols ${ }^{15}$, usando tertbutilhidroperóxido como sustrato, para evitar la participación en la reacción de la enzima catalasa, presente en la muestra. Una unidad de GPx en las condiciones experimentales se definió como aquella que cataliza la oxidación de $1 \mu \mathrm{mol}$ de glutatión por minuto. Los resultados se expresaron como mU/mg proteína.

La actividad de la enzima glutatión reductasa (GRd) se midió con el método de Smith y cols ${ }^{16}$. Una unidad de GRd en las condiciones usadas se definió como aquella que cataliza la reducción de $1 \mu \mathrm{mol}$ de DTNB a TNB por minuto. Los resultados se expresaron como $\mathrm{mU} / \mathrm{mg}$ proteína.

La actividad de la enzima $\gamma$-GCS se midió de acuerdo a Seelig y Meister ${ }^{17}$, monitorizando el cambio en absorbancia a $340 \mathrm{~nm}$ y $37^{\circ} \mathrm{C}$. La actividad específica se expresó como UI/mg proteína.

Niveles de glutatión total y oxidado. Se midieron utilizando el método espectrofotométrico de Griffith $^{18}$ en extractos ácidos de pulmón. La concentración de glutatión total se obtuvo mediante la interpolación de los resultados en una curva estándar. Para cuantificar los niveles de glutatión oxidado, las muestras fueron derivatizadas utilizando 2-vinilpiridina. La concentración de proteínas totales, se midió con el método de Bradford ${ }^{19}$, usando albúmina bovina como estándar.

Estadística. Los resultados se expresaron como promedio \pm 1 error estándar (EE). Se utilizó ANOVA y prueba de Tukey para comparar las diferencias en contenido de glutatión y en actividad enzimática a distintas edades y la actividad enzimática en distintos segmentos del pulmón. Se realizó estudio de correlación de Spearman entre actividad de GPx y GRd. Un valor de $p<0,05$ se consideró estadísticamente significativo ${ }^{20}$.

\section{RESUlTADOs}

Actividad de la enzima limitante en la síntesis de glutatión ( $\gamma$ GCS). El curso temporal de los cambios con la edad, en actividad de la enzima $\gamma$-GCS, se ilustra en la Figura 2. A los 7 días, esta actividad fue 


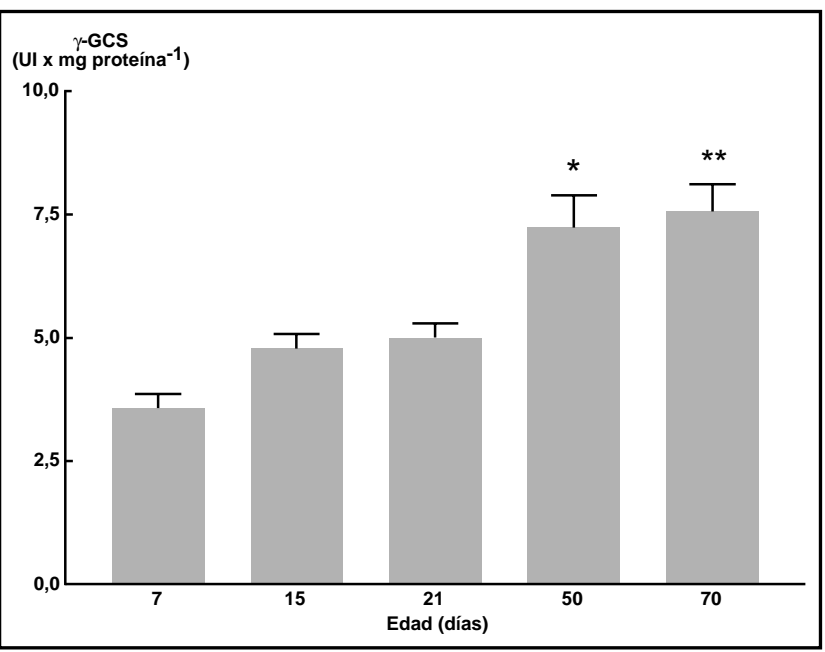

Figura 2. Actividad específica de la enzima $\gamma$-glutamilcisteína sintetasa ( $\gamma$-GCS) en el pulmón de la rata a distintas edades después del nacimiento. Las barras representan los promedios \pm 1 EE. *: $\mathrm{p}<0,05$; **: $\mathrm{p}<0,01$, con respecto al valor obtenido a los 7 días.

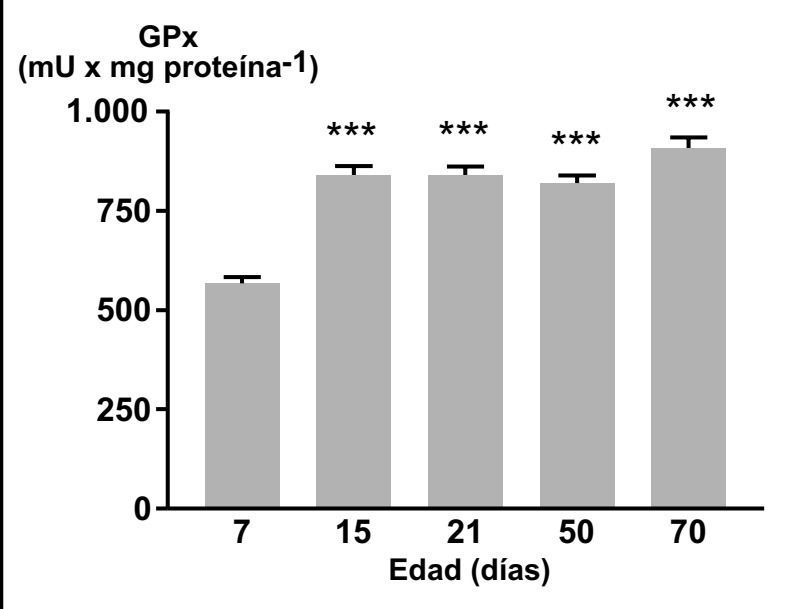

Figura 3. Actividad específica de la enzima glutatión peroxidasa (GPx) en el pulmón de la rata a distintas edades. Las barras representan los promedios $\pm 1 \mathrm{EE}$. ***: $\mathrm{p}<0,001$, con respecto al valor obtenido a los 7 días.

de 3,5 50 ,28 UI x mg proteína ${ }^{-1}$ y experimentó un aumento progresivo a lo largo del período estudiado. Si bien este aumento no alcanzó significancia estadística entre los 7 y los 21 días, los valores de actividad encontrados a los 50 y 70 días $(7,2 \pm 0,65$ y $7,6 \pm 0,56$ UI x mg prot. ${ }^{-1}$, respectivamente) fueron
$100 \%$ superiores a los observados a los 7 días ( $\mathrm{p}<0,05$ y $\mathrm{p}<0,01$, respectivamente).

Actividad de las enzimas del ciclo de óxido-reducción del glutatión

- Cambios dependientes de la edad en actividad específica de GPx y GRd: La Figura 3 muestra los valores de actividad de GPx en el pulmón a distintas edades después del nacimiento. A los 7 días, la actividad de esta enzima fue de $568 \pm 15$ mU x mg prot.-1, aumentó 32\% entre los 7 y los 15 días, alcanzando valores de $839 \pm 24 \mathrm{mU} \mathrm{x} \mathrm{mg} \mathrm{prot.}{ }^{-1}$ (p $<0,001)$ y se mantuvo igualmente elevada hasta la edad adulta (70 días).

Los valores de actividad de GRd se ilustran en la Figura 4. Esta actividad fue de $87,8 \pm 2,1 \mathrm{mU}$ x mg prot. ${ }^{-1}$ a los 7 días, aumentó $26 \%$ entre los 7 y los 15 días, alcanzando valores de 118 $\pm 3,6 \mathrm{mU}$ $x \mathrm{mg}$ prot. $^{-1}$ ( $\left.\mathrm{p}<0,001\right)$. Estos disminuyeron luego gradualmente, alcanzando a los 70 días, una reducción significativa con respecto al valor observado a los 15 días $(\mathrm{p}<0,001)$.

- Correlación entre actividad de GPx y GRd a distintas edades: Por participar estas enzimas en un ciclo de óxidoreducción, es importante conocer si la actividad de GPx se correlaciona con la actividad de GRd, para un adecuado funcionamiento del ciclo, dado que la cinética de los cambios dependientes de la edad es algo distinta para las dos enzimas. La Tabla 1 muestra que a cada una de las edades estudiadas existe una correlación positiva significativa entre las dos actividades. Sin embargo, la correlación fue mayor a los 7 y 70 días que en los tiempos intermedios, como se evidencia por el valor de $\mathrm{r}(\mathrm{r}=0,84$ y $\mathrm{r}=0,89$, respectivamente). La Figura 5 ilustra la correlación positiva significativa $(r=0,62, p<0,0001)$ que existe entre la actividad de GPx y de GRd al considerar todo el período de tiempo que incluyó el estudio (7 a 70 días).

- Distribución regional: La actividad de las enzimas del ciclo redox del glutatión en diferentes 
Figura 4. Actividad específica de la enzima glutatión reductasa (GRd) en el pulmón de la rata a distintas edades. Las barras representan los promedios \pm 1 EE. *: $p<0,05$ y ***: $p$ $<0,001$, con respecto al valor obtenido a los 7 días. \#. $\mathrm{p}<0,001$ con respecto al valor obtenido a los 15 días.

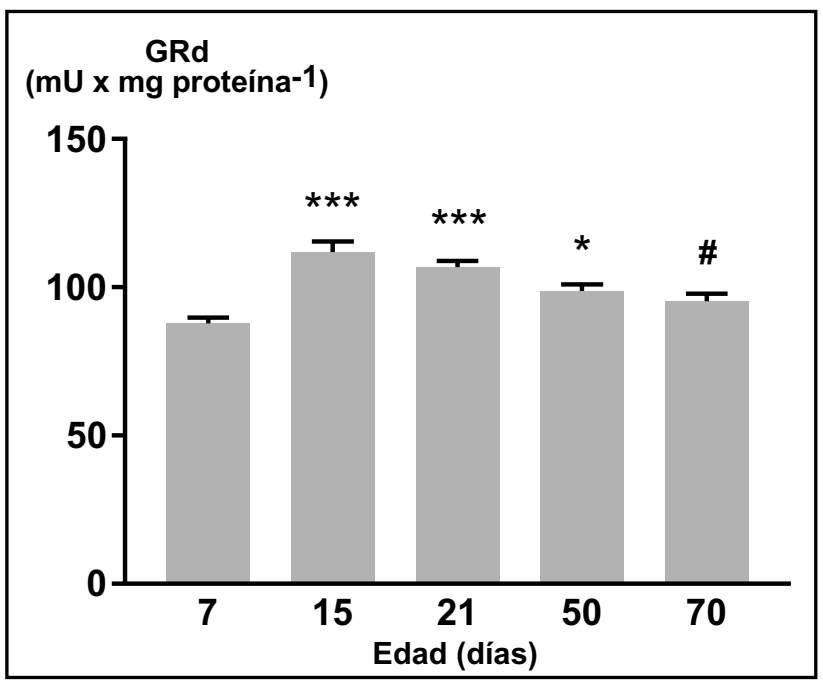

Tabla 1. Valores de $r$ y p para correlaciones entre G Px y G Rd en pulmones de ratas a distintas edades

\begin{tabular}{|lrrrrr|}
\hline & 7 días & 15 días & 21 días & 50 días & \multicolumn{1}{c|}{70 días } \\
\hline Valor $r$ & 0,84 & 0,75 & 0,51 & 0,68 & 0,89 \\
Valor $p$ & $<0,0001$ & $<0,01$ & $<0,01$ & $<0,0001$ & $<0,0001$ \\
\hline
\end{tabular}

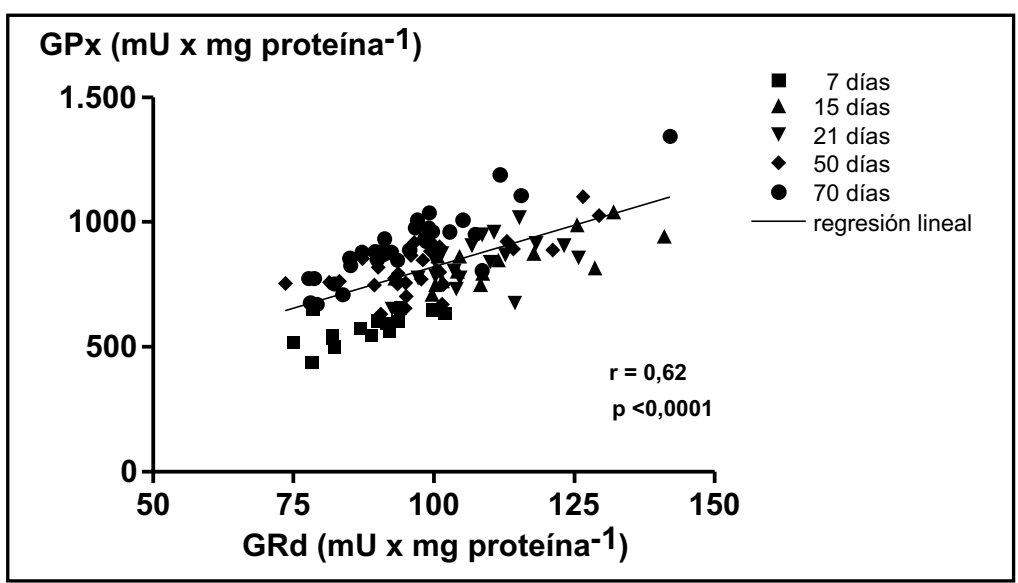

Figura 5. Correlación entre la actividad de la enzima glutatión peroxidasa (GPx) y la actividad de la enzima glutatión reductasa (GRd) entre los 7 y los 70 días de vida posnatal de la rata.

segmentos del pulmón de ratas de 50 y 70 días no mostró diferencias significativas, sugiriendo que la distribución de estas enzimas es homogénea en el pulmón.
Cambios dependientes de la edad en la concentración de glutatión total y oxidado. Los valores de concentración de glutatión total a distintas edades se ilustran en la Figura 6, que muestra que éste no 
se modificó significativamente en el período estudiado. El glutatión oxidado en cambio, presentó a edades tempranas, niveles cercanos a $7 \%$ del glutatión total, que cayeron a valores de $4 \%$ a los 21 días $(p<0,001)$ y se mantuvieron en ese nivel hasta el final del período estudiado (Figura 7).

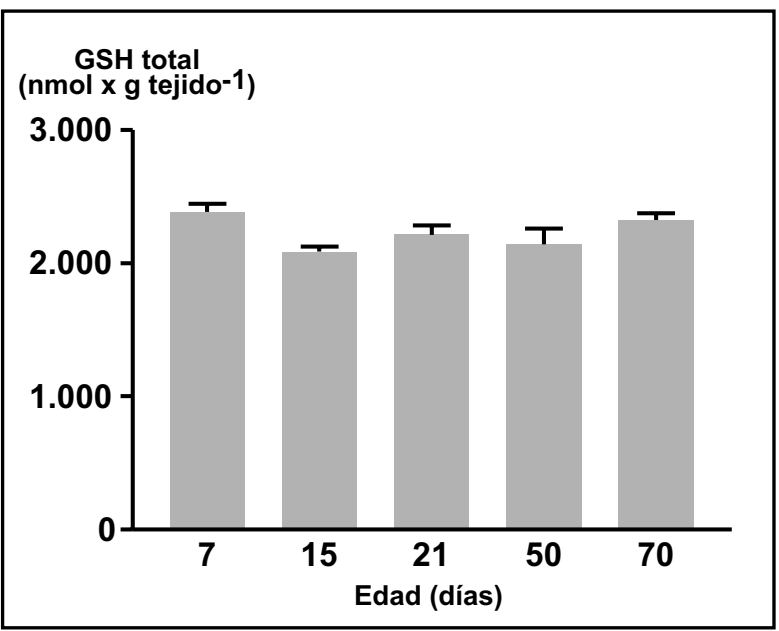

Figura 6. Concentración de glutatión total en el pulmón de la rata a distintas edades después del nacimiento. Las barras representan los promedios $\pm 1 \mathrm{EE}$.

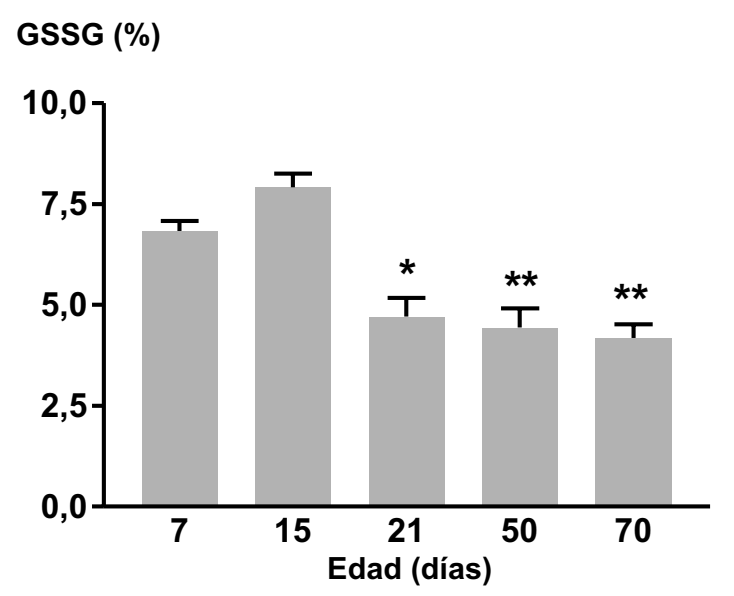

Figura 7. Glutatión oxidado (GSSG) expresado como porcentaje del glutatión total en el pulmón de la rata a distintas edades después del nacimiento. *: $p<0,05$; ** $p$ $<0,01$ en relación a valores obtenidos a los 7 y 15 días.

\section{Discusión}

El hallazgo en pulmones de seres humanos adultos de una mayor actividad de la enzima superóxido dismutasa, en comparación con pulmones de recién nacidos, y en éstos en comparación con pulmones de fetos ${ }^{21}$, contribuyó a plantear la posibilidad de la existencia de una regulación de las enzimas antioxidantes durante el desarrollo y posterior crecimiento del pulmón de los mamíferos. Los resultados de nuestro estudio apoyan dicha hipótesis, ya que las enzimas que participan en el metabolismo del glutatión experimentan un aumento en su actividad durante el desarrollo posnatal del pulmón de la rata, con un curso temporal propio de cada enzima, manteniéndose constante el contenido de glutatión total.

Nuestro trabajo, en parte, confirma los resultados del estudio de Yam y cols en pulmones de ratas $^{10}$, que mostró un aumento en la actividad de las enzimas del ciclo de óxido-reducción del glutatión y de otras enzimas antioxidantes desde antes del nacimiento y durante el período posnatal. Sin embargo, dicho estudio, a diferencia del nuestro, mostró un aumento en el contenido de glutatión durante el período posnatal, sin evaluar su grado de oxidación ni su síntesis. Nuestro estudio, además de confirmar el aumento posnatal en la actividad de las enzimas del ciclo redox del glutatión (GPx y GRd), muestra una correlación positiva significativa entre las actividades de ambas enzimas en el período estudiado, que sugiere un adecuado reciclaje del GSH, acompañado de un aumento en la actividad de la enzima limitante de la síntesis del glutatión, con conservación de los niveles de glutatión reducido.

En relación con el desarrollo posnatal del pulmón de la rata, se describen tres etapas claramente diferentes ${ }^{11-13}$ : a) una etapa de expansión pulmonar que abarca los 4 primeros días de vida, b) una etapa de proliferación celular con formación de tabiques alveolares, entre los 4 y 13 días de vida y c) un período de remodelación de los tabiques, en que los capilares alveolares adoptan la disposición propia del pulmón del animal adulto, desde el día 13 en adelante. De acuendo a esto, el significativo aumento en la actividad de las enzimas del 
ciclo de óxido-reducción del glutatión se produce antes de los 15 días de vida, período que coincide con la mayor actividad metabólica propia de la formación de nuevos alvéolos. Durante este período encontramos además, niveles leves pero significativamente elevados de glutatión oxidado. El aumento en la actividad de la enzima $\gamma$-GCS en cambio, se produce más tardíamente, una vez terminada la fase de proliferación celular propia de la formación de nuevos tabiques alveolares y es de mayor magnitud que el aumento en la actividad de las enzimas del ciclo redox.

$\mathrm{Si}$ bien las señales involucradas en el aumento de actividad de las enzimas antioxidantes son desconocidas, Frank y cols han propuesto que el aumento de sus sustratos en condiciones de estrés oxidativo, constituiría el principal mecanismo responsable del aumento en la actividad enzimática ${ }^{22}$. Al respecto, se sabe que la actividad de GPx está regulada por la concentración de sus sustratos $\left(\mathrm{H}_{2} \mathrm{O}_{2}\right.$, hidroperóxidos y distintos peróxidos lipídi$\cos )^{22}$, mientras que los niveles de GSSG, posiblemente en respuesta a una mayor actividad de GPx, regulan la actividad de GRd. Los mayores niveles de glutatión oxidado (GSSG) encontrados en nuestro estudio, en la etapa temprana del desarrollo posnatal del pulmón, sugieren que el aumento en la actividad de las enzimas del ciclo redox se podría producir en respuesta a estrés oxidativo al que es sometido el pulmón en dicho período. Este estrés oxidativo podría estar relacionado tanto con la propia proliferación celular en la etapa de formación de los alvéolos, como con la exposición del pulmón a distintas noxas propias del ambiente extrauterino, entre ellas, la hiperoxia relativa.

Por otra parte, el aumento en la actividad enzimática puede obedecer también a un aumento en la síntesis de nuevas enzimas, ya que el estado redox celular y particularmente la relación GSSG/ GSH total, participan en la activación de factores de transcripción como AP-1 o NF- $\kappa \Omega^{23,24}$ que regulan la expresión de enzimas antioxidantes. Sin embargo, Clerch y Massaro encontraron que al menos para la enzima GPx, el aumento en actividad

\section{REFERENCIAS}

1. Cross CE, Van Der Vliet A, O’Neill CA, Eiserich JP. Reactive oxygen species and the lung. Lancet 1994; 334: 930-3. observado en el período prenatal y posnatal temprano de la rata, no se acompaña de un aumento en la concentración de $\mathrm{ARNm}^{25}$ y se debería a fenómenos postranscripcionales, como por ejemplo, cambios conformacionales de la enzima, relacionados o no con la disponibilidad de selenio, indispensable para la actividad enzimática ${ }^{26}$.

Dado que los fenómenos regulatorios de las defensas antioxidantes durante el período posnatal no se limitan sólo al pulmón y han sido descritos también en otros tejidos ${ }^{27,28}$, es posible especular que la respuesta adaptativa dependiente de la edad observada en el pulmón podría responder a factores sistémicos.

Por otra parte, nuestros resultados muestran que la distribución de la actividad enzimática del ciclo redox del glutatión pulmonar es homogénea, lo que permite validar y comparar trabajos realizados con muestras de diferentes zonas del pulmón.

En resumen, el sistema del glutatión en el pulmón de la rata experimenta importantes cambios fisiológicos en el período posnatal, los que involucran a diversas enzimas que participan en su metabolismo. Es posible que el pulmón humano experimente cambios similares en el metabolismo del glutatión, ya que, al igual que en la rata y otros mamíferos, su desarrollo no está completo al momento del nacimiento y alrededor de $80 \%$ del desarrollo alveolar se produce después de nacer $^{13}$. De ser así, es dable esperar que la sensibilidad de las células del pulmón frente a agentes injuriantes ambientales, sea completamente distinta durante el desarrollo que durante la vida adulta, haciéndose necesario al igual que en la rata, tomar en consideración la regulación fisiológica, al momento de evaluar los cambios en antioxidantes pulmonares frente a distintos agentes que dañan el pulmón en diferentes etapas de la vida. La maduración incompleta de los sistemas antioxidantes de las células pulmonares podría constituir un mecanismo de daño de las células pulmonares en rápida proliferación al ser expuestas a contaminantes ambientales, con posibles efectos a largo plazo en la función pulmonar del adulto.

2. Rahman I, Macnee W. Oxidative stress and regulation of glutathione in lung inflammation. Eur Respir J 2000; 16: 534-54.

3. Снавот J, Mitchell JA, Gutteridge JMC, Evans TW. Reactive oxygen species in acute 
lung injury. Eur Respir J 1998; 11: 745-57.

4. Chow CW, Herrera Abreu MT, Suzuki T, Downey GP. Oxidative stress and acute lung injury. Am J Respir Cell Mol Biol 2003; 29: 427-31.

5. KaARTeenaho-Wiik R, Kinnula VL. Distribution of antioxidant enzymes in developing human lung, respiratory distress syndrome, and bronchopulmonary dysplasia. J Histochem Cytochem 2004; 52: 1231-40.

6. Frank L, Groseclose EG. Preparation for birth into an $\mathrm{O}_{2}$-rich environment: the antioxidant enzymes in the developing rat lung. Pediatr Res 1984; 18 : 240-4.

7. Frank L, Sosenko IRS. Prenatal development of lung antioxidant enzymes in four species. J Pediatr 1987; 100: 106-10.

8. Tanswell AK, Freeman BA. Pulmonary antioxidant enzyme maturation in fetal and neonatal rat. I. Developmental profiles. Pediatr Res 1984; 18: 584-97.

9. Gerdin E, Tyden O, ERiKsson UJ. The development of antioxidant defense in the perinatal rat lung: activities of superoxide dismutase, glutathione peroxidase, and catalase. Pediatr Res 1985; 19: 687-91.

10. YAm J, Frank L, RobeRTS RJ. Age-related development of pulmonary antioxidant enzymes in the rat. Proc Soc Exp Biol Med 1978; 157: 293-6.

11. Burri PH, Dbaly J, Weibel ER. The postnatal growth of the rat lung I. Morphometry. Anat Rec 1974; 178: 711-30.

12. BuRRI PH. The postnatal growth of the rat lung I. Morphology. Anat Rec 1974; 180: 77-98.

13. PinkeRTon KE, JoAd JP. The mammalian respiratory system and critical windows of exposure for children's health. Environ Health Perspect 2000; 108 (suppl 3): 457-62.

14. Cantin AM, North SL, Hubbard RC, Crystal RG. Normal alveolar epithelial lining fluid contains high levels of glutathione. J Appl Physiol 1987; 63: 152-7.

15. Gunzier WA, Kramer S, Flohé L An improved coupled test procedure for glutathione peroxidase in blood. Z Klin Chem Klin Biochem 1974; 12: 444-8.

16. Smith IK, Vier Heuler TL, Thorne CA. Assay of glutathione reductase in crude tissue homogenates using 5,5-dithiobis (2-nitrobenzoic acid). Analytical Biochemistry 1988; 75: 408-13.
17. Seelig GF, Meister A. Glutathione biosynthesis: gamma-glutamylcysteine synthetase. Methods Enzymol 1985; 113: 379-90.

18. GRIFFTH O. Determination of glutathione and glutathione disulfide using glutathione reductase and 2-vinilpyridine. Anal Biochem 1980; 106: 207-12.

19. BRADFORD MM. A rapid and sensitive method for the quantitation of microgram quantities of protein utilizing the principle of protein dye. Anal Biochem 1976; 72: 248-54.

20. SoкаL RR, Rohlғ FJ. Eds. Biometry. New York: W.H. Freeman and Company, 1981.

21. AUtOR AP, FRANK L, ROBERTS RJ. Developmental characteristics of pulmonary superoxide dismutase: relationship to idiopathic respiratory distress syndrome. Pediatr Res 1976; 10: 154-8.

22. Frank L, Price LT, Whitney PL Possible mechanism for late gestational development of the antioxidant enzymes in fetal rat lung. Biol Neonate 1996; 70: 116-27.

23. Cho S, Urata Y, Lda T, Goto S, Yamaguchi M, Sumikawa K, Kondo T. Glutathione downregulates the phosphorilation of I-Kß: autoloop regulation of the NF-Kß mediated expression of NF-Kß subunits by TNF-A in mouse vascular endothelial cells. Biochem Biophys Res Commun 1998; 253: 104-8.

24. Meyer M, Schreck R, Bauerie PA. Hydrogen peroxide and antioxidants have opposite effects on activation of NF-Kß and AP-1 in intact cells: AP-1 as secondary antioxidant-responsive factor. EMBO J 1993; 12: 2005-15.

25. Clerch LB, Massaro D. Rat lung antioxidant enzymes: differences in prenatal gene expression and regulation. Am J Physiol 1992; 263: L466-L470.

26. ClerRCH LB, Massaro DJ. Lung antioxidant enzyme gene expression and tolerance to hyperoxia. En: Clerch LB y Massaro DJ, ed. Oxygen, gene expression, and cellular function. New York: Marcel Dekker Inc, 1997; 399-424.

27. Montecinos L, Ramirez B, Lisboa C, Borzone G. Metabolismo de peróxidos en músculos respiratorios: efecto del crecimiento, desarrollo, y envejecimiento. Rev Méd Chile 1999; 127: 269-75.

28. Harman AW, MckenNa M, Adamso N GM. Postnatal development of enzyme activities associated with protection against oxidative stress in the mouse. Biol Neonate 1990; 57: 187-93. 